\title{
Impact of Social Status and Antidepressant Treatment on Neurogenesis in the Baboon Hippocampus
}

\author{
Melody V Wu ${ }^{1,2}$, Jul Lea Shamy ${ }^{3,8}$, Gillinder Bedi ${ }^{1,4}$, Chien-Wen J Choi ${ }^{1,5}$, Melanie M Wall ${ }^{1,5}$, \\ Victoria Arango ${ }^{1,6}$, Maura Boldrini ${ }^{1,6}$, Richard W Foltin ${ }^{1,4}$ and René Hen*,1,2,7 \\ 'Department of Psychiatry, Columbia University, New York, NY, USA; 'Division of Integrative Neuroscience, New York State Psychiatric Institute, \\ New York, NY, USA; ${ }^{3}$ Department of Neuroscience and Friedman Brain Institute, Mt Sinai School of Medicine, New York, NY, USA; ${ }^{4}$ Division on \\ Substance Abuse, New York State Psychiatric Institute, New York, NY, USA; ${ }^{5}$ Division of Biostatistics, New York State Psychiatric Institute, \\ New York, NY, USA; ${ }^{6}$ Division of Molecular Imaging and Neuropathology, New York State Psychiatric Institute, New York, NY, USA; \\ ${ }^{7}$ Departments of Neuroscience and Pharmacology, Columbia University, New York, NY, USA
}

Adult hippocampal neurogenesis is critically implicated in rodent models of stress and anxiety as well as behavioral effects of antidepressants. Whereas similar factors such as psychiatric disorder and antidepressant administration are correlated with hippocampal volume in humans, the relationship between these factors and adult neurogenesis is less well understood. To better bridge the gap between rodent and human physiology, we examined the numbers of proliferating neural precursors and immature cells in the hippocampal dentate gyrus (DG) as well as in vivo magnetic resonance imaging (MRI)-estimated whole hippocampal volume in eight socially dominant- or subordinate-like (SL) baboons administered the antidepressant fluoxetine or vehicle. SL baboons had lower numbers of proliferating cells and immature neurons than socially dominant-like baboons. Fluoxetine treatment was associated with a larger whole hippocampal volume but surprisingly resulted in lower numbers of immature neurons. These findings are the first to indicate that adult neurogenesis in the baboon hippocampal DG may be functionally relevant in the context of social stress and mechanisms of antidepressant action.

Neuropsychopharmacology (2014) 39, I86I-187I; doi:I0.1038/npp.2014.33; published online 5 March 20|4

Keywords: adult neurogenesis; dentate gyrus; antidepressants; social stress

\section{INTRODUCTION}

The generation of new neurons in the adult mammalian brain, although once controversial, is now widely accepted and acknowledged to be involved in a variety of processes (Ming and Song, 2011). Specifically, adult neurogenesis in the subgranular zone (SGZ) of the mammalian hippocampal dentate gyrus (DG) has been implicated in functions ranging from learning and memory to anxiety-related behaviors (Deng et al, 2010; Sahay and Hen, 2007). In rodents, ablation of adult neurogenesis results in deficits in contextual discrimination, novelty recognition, and spatial memory (Denny et al, 2012; Sahay et al, 2011) as well as blunted behavioral responses to antidepressants (David et al, 2009; Surget et al, 2008). Consistent with this, chronic exposure to stressful events, elevated levels of corticosterone, and stimulus-poor environments all lead to a decrease in overall levels of neurogenesis in rodents (Cameron and Gould, 1994; Dranovsky

*Correspondence: Professor R Hen, Center for Neurobiology and Behavior, Columbia University, I05I Riverside Drive, Unit 87, Kolb Annex, Room 767, New York, NY 10032, USA, Tel: + I 212646 774 7108, Fax: + I 212543 5074, E-mail: rh95@columbia.edu ${ }^{8}$ Current address: Pfizer, Neuroscience, Cambridge, MA 02139, USA Received 2 October 20 I3; revised 2 January 20 I4; accepted 20 January 2014; accepted article preview online 12 February 2014 et al, 2011; Pham et al, 2003). By contrast, environmental enrichment and antidepressant administration result in increased numbers of adult-born neurons (Dranovsky et al, 2011; Kempermann et al, 1997; Malberg et al, 2000).

Whereas most studies on adult hippocampal neurogenesis have been conducted in rodents, neurogenesis in higher mammals has recently received increased attention. The classic paper demonstrating neurogenesis in the human adult hippocampus (Eriksson et al, 1998) has been complemented by studies examining expression of endogenous markers of immature neurons (Boldrini et al, 2012; Boldrini et al, 2009; Knoth et al, 2010; Lucassen et al, 2010; Monje et al, 2007). Adult neurogenesis in the DG of nonhuman primates has also been shown, primarily in the macaque species (Gould et al, 1998; Kohler et al, 2011; Kornack and Rakic, 1999; Perera et al, 2011). However, the number of proliferating and maturing cells are thought to be lower in primates than rodents (Kornack and Rakic, 1999), to the point that some have suggested a significantly reduced functional role of neurogenesis in primates compared with rodents and birds (Rakic, 2002). Of note, however, is recent work suggesting that even low numbers of proliferating cells may still result in considerable accumulation of adult-born neurons in the DG over the human lifespan (Spalding et al, 2013). 
Despite the apparent reduction in level, adult neurogenesis in the primate hippocampus appears to be regulated by external factors in a manner similar to that in rodents. Social stress reduces the number and maturation of hippocampal neurogenic precursors in monkeys, an effect rescued by antidepressant administration (Gould et al, 1998; Perera et al, 2011). In addition, altered rates of neurogenesis have been hypothesized to underlie some of the changes in hippocampal volume seen in several psychiatric disorders and following antidepressant treatment in humans (Czeh and Lucassen, 2007). Patients with major depressive disorder (MDD) treated with selective serotonin reuptake inhibitors have a larger DG and whole hippocampal volume compared with untreated patients and controls (Boldrini et al, 2009; Huang et al, 2013), which may be explained in part by an increase in both neurogenesis and angiogenesis (Boldrini et al, 2012). However, a definitive relationship between hippocampal volume and adult neurogenesis and how each of these measures is modulated by antidepressants or stress, whether concomitantly or exclusively, has yet to be established. Understanding the features of adult hippocampal plasticity in non-human primates is an important step in bridging the gap between what is known in rodents and what needs to be elucidated in human physiology and pathology.

We thus examined adult hippocampal neurogenesis in an evolutionarily higher order primate with a larger brain than the macaque, the baboon. Utilizing markers of proliferating cells and immature neurons, we examined the DG of both dominant-like (DL) and subordinate-like (SL) young adult, male baboons following chronic administration of fluoxetine or vehicle. We sought to determine whether the extent and rate of neuronal production is altered by social experience or antidepressant administration, and whether cellular changes reflect alterations in overall hippocampal volume as estimated by magnetic resonance imaging (MRI).

\section{MATERIALS AND METHODS}

\section{Animals}

Eight young adult, post-pubertal (10-12 years old), male baboons (Papio cynocephalus anubis with an average lifespan of 30 years in the wild) were individually housed and maintained on a 12:12 light cycle. Water and pellet food were available ad libitum, along with daily fruits and vegetables. All procedures complied with the NIH AALAC and NYSPI IACUC guidelines. Animals had previously participated in feeding and drug addiction studies (Foltin, 2011). Briefly, all subjects were tested in the same series of behavioral pharmacology experiments on feeding behavior 4 years before. During that time, all subjects equally received 100 injections of low doses of drugs designed to affect food intake (dexfenfluramine and amphetamine), could work to earn a highly palatable sweet candy on 120 days, and could smoke a low dose of methamphetamine on 120 different days. In total, they all received drug on up to 220 of the 1340 days they were in studies. All baboons were drug-free for about 3 months before the current study and had received $\sim 36$ drug doses in the 9 months prior.

\section{Assessing Social Status}

Social status was independently assessed by three individuals with nearly 60 years combined experience working with singly housed baboons and other monkeys with visual and olfactory access to conspecifics. Baboons in the wild live in complex social environments and even when singly housed display behaviors that parallel social behaviors observed in the wild. While behavior would clearly vary if the baboons were allowed to live in a large social unit, our estimates of social status thus provide a model of real world behavior. Briefly, baboons were rated as either DL or SL within the colony based on the number of dominant and subordinate behaviors exhibited as described below. Dominant behaviors include the following: (1) readily reaching out of the cage to grab a caretaker or take a food treat; (2) reaching into the neighboring cage; (3) spending increased time at the front of the cage and presenting either chests or flanks for grooming; (4) throwing items (toys, uneaten food) out of the cage; (4) initiation of vocalizations including 2-stage barks and grunts associated with feeding and socialization; (5) staring at conspecifics as a manifestation of a threat; (6) cage rattling; (7) readily interacting with novel objects or food items; (8) ending grooming periods with a brief aggressive display; and (9) yawning and displaying their canines or lip-smacking and teeth-chattering when approached by a caretaker. Subordinate behaviors include (1) not reaching out of the cage or barely doing so even to take treats; (2) hesitation to come to the cage front; (3) responses to staring or cage rattling but rare initiation of such behavior; (4) not readily interacting with novel objects or food items; and (5) fleeting presentation of their buttocks to caretakers or conspecifics then walking away after initial contact.

Each of the three experienced raters created a rankordering of the eight baboons from most DL to most SL based on the behaviors listed above with the most DL baboons only displaying dominant behaviors, the most SL animals displaying only subordinate behaviors, and the intermediate baboons displaying some of both types of behavior. Initial estimates of dominance were made when the baboons were first moved into the colony room together. Knowing their status helped investigators interact with each baboon in a way that minimized stress-for example,more slowly approaching SL animals while avoiding direct eye contact. Social status was periodically reassessed and remained stable over time. Status was last assessed immediately before assigning baboons to experimental condition for the present study.

\section{Fluoxetine}

Fluoxetine hydrochloride powder (Dr. Reddy's Laboratories, Bridgewater, NJ) was placed in two sun-dried figs and given at 8:45 am daily. Dose was increased over 5 days from $1-5 \mathrm{mg} / \mathrm{kg} /$ day and subsequently maintained for 30 days, at which point animals were perfused. Vehicle consisted of a similar weight of dried powdered milk in two figs. Four baboons (two DL and two SL) received vehicle and four (two DL and two SL) received fluoxetine. Post-mortem hepatic fluoxetine and norfluoxetine levels were confirmed with liquid chromatography with fluorescence detection as described previously (Suckow et al, 1992). 


\section{BrdU Infusions and Perfusions}

Beginning 5 days before perfusion, animals were given daily intravenous infusions of $100 \mathrm{mg} / \mathrm{kg} 5$-bromo-2'-deoxyuridine (BrdU; Roche, Basel, Switzerland) dissolved in normal saline while under isoflurane following initial ketamine anesthesia. Two hours after the last BrdU infusion, animals were anesthetized with ketamine followed by an overdose of sodium pentobarbital and perfused transcardially with heparinized normal saline followed by $4 \%$ cold paraformaldehyde. Brains were removed and postfixed overnight before cryoprotection in $30 \%$ sucrose. The right hippocampus was then blocked, sectioned at $40 \mu \mathrm{m}$ on a freezing microtome (Microm HM440E), collected in cryoprotectant (30\% ethylene glycol in $0.1 \mathrm{M}$ phosphate buffer), and stored at $-20^{\circ} \mathrm{C}$.

\section{Histology}

Every 10th section was immediately slide-mounted at the time of sectioning and stained with cresyl violet to assess the volume of the granule cell layer (GCL) of the DG. Nisslstained sections were aligned to a baboon neuroanatomical reference atlas (Davis and Huffman, 1968) and provided an anatomic reference in order to align immunofluorescencestained sections along the rostrocaudal axis of the hippocampus in each animal. Slides were imaged on an AxioObserver A.1 (Zeiss) at $\times 2.5$ magnification. Using Image (NIH), images were made binary and thresholded to extract the volume of the GCL of the DG.

Every 40th section was stained for either BrdU or doublecortin (DCX) in combination with neuronal nuclei (NeuN) using standard free-floating immunofluorescence procedures. BrdU staining was preceded by antigen retrieval in $2 \mathrm{~N} \mathrm{HCl}$ at $65^{\circ} \mathrm{C}$ followed by neutralization in $0.1 \mathrm{M}$ boric acid at room temperature. Primary antibodies were mouse anti-NeuN (1:300; Chemicon, Temecula, CA), goat antiDCX (1:500; Santa Cruz Biotechnology, Dallas TX), and rat anti-BrdU (1:100; Serotec, Raleigh, NC). Secondary antibodies were Alexa-Fluor 488-donkey anti-mouse $(1: 300$; Invitrogen, Carlsbad, CA), Cy3-donkey anti-goat $(1: 800$; Jackson ImmunoResearch, West Grove, PA), and Cy3donkey anti-rat $(1: 800$; Jackson ImmunoResearch). Sections were exposed to primary antibodies for $44-46 \mathrm{~h}$ at $4{ }^{\circ} \mathrm{C}$ and secondary antibodies for $2 \mathrm{~h}$ at room temperature. BrdU- and DCX-stained sections were imaged on an Olympus FluoView confocal microscope at $\times 20$ magnification. Z-stacks $(3.65 \mu \mathrm{m}$ thick) were acquired and all BrdUand DCX-positive cells were enumerated through the rostrocaudal extent of the SGZ of the DG. The number of enumerated cells was then multiplied by 40 to obtain an estimate of the total number of BrdU- and DCX-positive cells present. Maturity of DCX-positive cells was characterized based on the degree of dendritic branching (or number of intersection points). Specifically, DCX-positive cells were classified as either having (1) tertiary or higher-order dendrites or (2) secondary or lower-order dendritic branching (Wang et al, 2008). All DCX-positive cells were also traced using the 'Simple Neurite Tracer' plugin within the Fiji platform (Longair et al, 2011; Schindelin et al, 2012) to obtain measures of total dendritic length, number of dendritic branches, and average branch length per cell for each animal.

\section{MRI}

Between 5 and 7 days before perfusion, animals were anesthetized with ketamine followed by propofol, and imaged in the NYSPI MRI core facility. High-resolution $\mathrm{T}$-1 weighted structural (3-Dimensional Inversion Recovery Fast Spoiled Gradient Recalled Echo; 3D IR-FSPGR) images were acquired in the coronal plane on a 3-Tesla GE Signa scanner (Inversion time $=450 \mathrm{~ms} ; \mathrm{TE}=1.7 \mathrm{~ms}$; flip angle $=14$ degrees; $\mathrm{FOV}=14 \mathrm{~cm}^{2}$; acquisition matrix $=256$ $\times 160$; slice thickness $=1 \mathrm{~mm}$; number of excitation $(\mathrm{NEX})=2$; voxel size $=0.55 \times 0.88 \times 1 \mathrm{~mm})$.

Image processing was undertaken on a MacbookPro using the software package Analyze 10.0 (AnalyzeDirect, Overland Park, KS) for manual tracing and on a 12-core MacPro using Automated Normalization Tools (ANTs; http://picsl.upenn.edu/ANTS/; UPenn, Philadelphia, PA; Avants et al, 2008; Avants et al, 2011) for automated template generation, registration, and segmentation. Automated protocols used in this baboon study were modeled after previous human studies demonstrating superior performance and reliability of ANT template-driven segmentation over other segmentation methods (Avants et al, 2010).

First, an adult male baboon template MRI was generated by iteratively using the antsRegistration and shape update tools in ANTs. The brain was then manually delineated on the template MRI in Analyze 10.0 (Figure 4a). The template MRI and brain mask were then used to extract the brain, and a refined brain template was generated using ANTs. Baboon neuroanatomical references (Davis and Huffman, 1968) and tracing protocols developed for rhesus monkeys (Shamy et al, 2006; Shamy et al, 2011) were then used to guide tracing of the hippocampus on the baboon brain template (Figure $4 \mathrm{~b}$ and $\mathrm{c}$ ). The hippocampus was defined inclusive of the DG, CA1-3 fields of the hippocampus, subiculum, presubiculum, and parasubiculum. Additionally, the hippocampus was segmented into 10 similarly spaced hippocampal regions along the rostrocaudal axis (Figure 4d). The group brain template was used to segment the individual subject data for the hippocampus. Finally, template-based segmentation along the rostrocaudal axis of the hippocampus was completed on the resulting hippocampal region. All voxels within the boundaries were included in the hippocampal volumes calculated in the subject MRI space, not in the template space.

\section{Statistical Analyses}

All quantification was performed with investigators blinded to social status and drug treatment. Between-subject effects were first assessed with two-way analyses of variance (ANOVAs) with social status as one factor and drug treatment as the second. As no significant interactions were observed, main effect regression without interactions was performed and presented in the text. Differential effects along the rostrocaudal axis were assessed using a three-way repeated measures ANOVA (with social status and drug treatment as between-subject factors, section number as the repeated measure, and two-way interactions between section number and social status or drug treatment). Post hoc tests were performed in the case of significant interactions to identify which sections differed. As the 

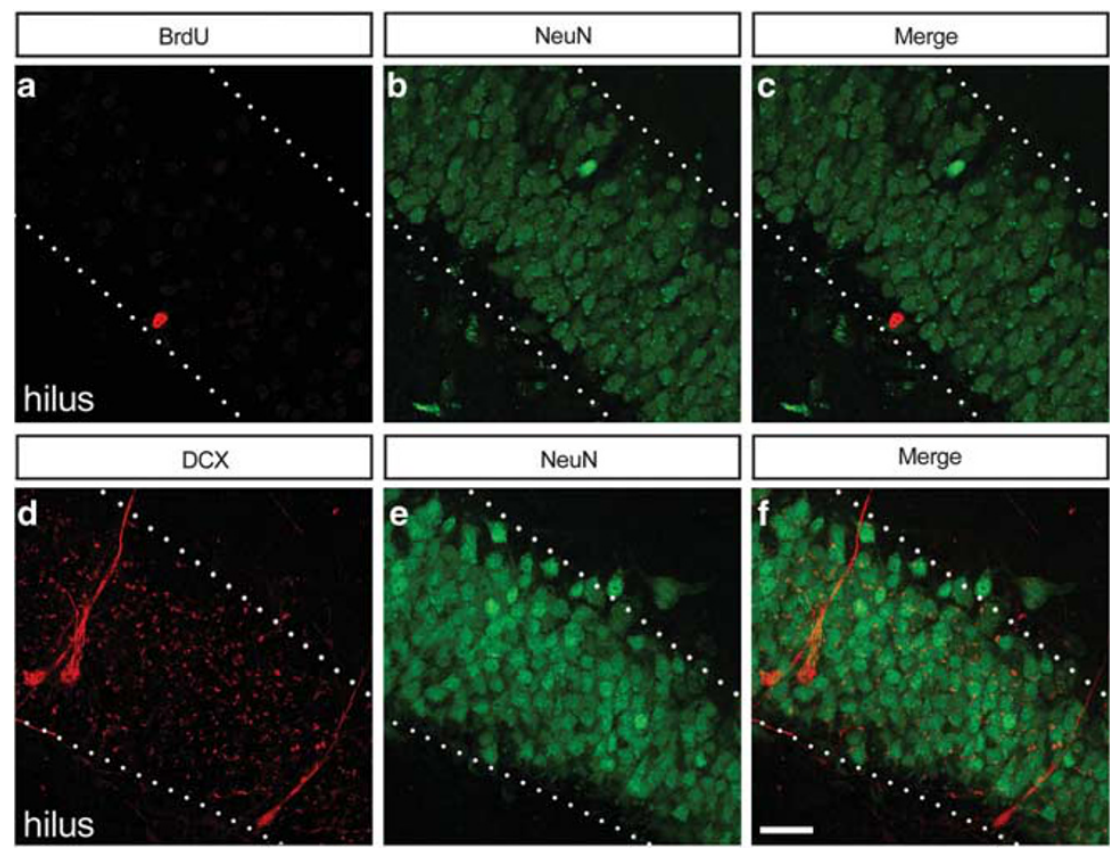

Figure I Markers of proliferating cells and immature neurons in the adult baboon dentate gyrus. Representative coronal sections through an adult, male baboon hippocampus stained for neuronal nuclei (NeuN) and either bromodeoxyuridine (BrdU) (a-c) or doublecortin (DCX) (d-f). (a-c) BrdU-positive cells can be visualized in the subgranular zone following 5 days of daily BrdU infusion, and do not colocalize with NeuN-positive cells. ( $d-f$ ) Cell bodies of DCX-positive cells are localized in the inner third of the granule cell layer and extend processes away from the hilus toward the molecular layer of the dentate gyrus. Scale bar equals $25 \mu \mathrm{m}$. Hilus is to the lower left of each image and dotted lines circumscribe the granule cell layer.

small sample size makes it difficult to test for violations of normality and other assumptions in ANOVA, a nonparametric procedure was also used to analyze the data, given its robustness to violations of distributional assumptions. The rank transformation approach assigns ranks to observations before applying a conventional parametric procedure (Conover and Iman, 1981). In this case, the entire set of observations was ranked from smallest to largest, and average ranks were computed in case of ties. Two- and three-way repeated measures ANOVAs (as above) were then applied to the ranks. Results were deemed statistically significant if both the parametric and non-parametric $p$-values were $<0.05$ (Supplementary Table S1).

\section{RESULTS}

\section{Proliferation and Neurogenesis in the Baboon DG}

To assess the extent of cell proliferation in the hippocampus of the baboon, we examined incorporation of the thymidine analog, BrdU, a marker of DNA synthesis, in the DG of adult, post-pubertal males. Immediately following 5 days of daily intravenous BrdU infusion (Kornack and Rakic, 1999), proliferating cells were detected in the brains of all animals. Owing to the fact that BrdU is incorporated in all dividing cells, and thus an insufficient marker of neural progenitor cells, we reduced the likelihood of overrepresentation by limiting quantification of BrdU-positive cells to the inner third of the GCL, corresponding to the SGZ of the DG. In the SGZ, BrdU-positive cells exhibited the classic rounded cell body morphology of granule cell precursors (Figure 1a-c). As expected, BrdU expression did not colocalize with expression of the mature neuronal marker, $\mathrm{NeuN}$, indicat- ing that 5 days were insufficient for the newborn cells to differentiate into mature neurons.

As our BrdU detection protocol reveals only proliferation and neither survival nor differentiation of the newly generated cells, we also examined expression of the endogenous microtubule-associated protein, DCX, to further assess the extent of adult hippocampal neurogenesis. DCX is used as a marker of immature neurons routinely in rodents (Couillard-Despres et al, 2005; Wang et al, 2008), and more recently in human and non-human primates (Knoth et al, 2010; Kohler et al, 2011). DCX is transiently expressed during adult neurogenesis, and downregulation of DCX occurs at the same time during which cells begin to express NeuN (Couillard-Despres et al, 2005). To minimize the likelihood of counting DCX-positive cells that are not representative of adult-born immature neurons (Knoth et al, 2010; Rakic, 2002), only cells located along the SGZ with a visible process extending from the soma toward the molecular layer of the DG were quantified (Figure 1d-f). BrdU- and DCX-positive cells in the SGZ were quantified in every 40 th $(40 \mu \mathrm{m}$ thick) section of the entire rostrocaudal extent of the DG in each animal (Figure 2, Supplementary Figure S1a-d). The number of quantified cells was then multiplied by 40 to determine the estimated total number of BrdU- and DCX-positive cells in the SGZ. We observed similar numbers of proliferating cells in the DG as reported in other primate species (Gould et al, 1998; Kohler et al, 2011; Kornack and Rakic, 1999).

Social Status, Antidepressant Administration, and Adult Hippocampal Neurogenesis

As neurogenesis in the adult DG can be regulated by social stress or status in both rodents and non-human primates 

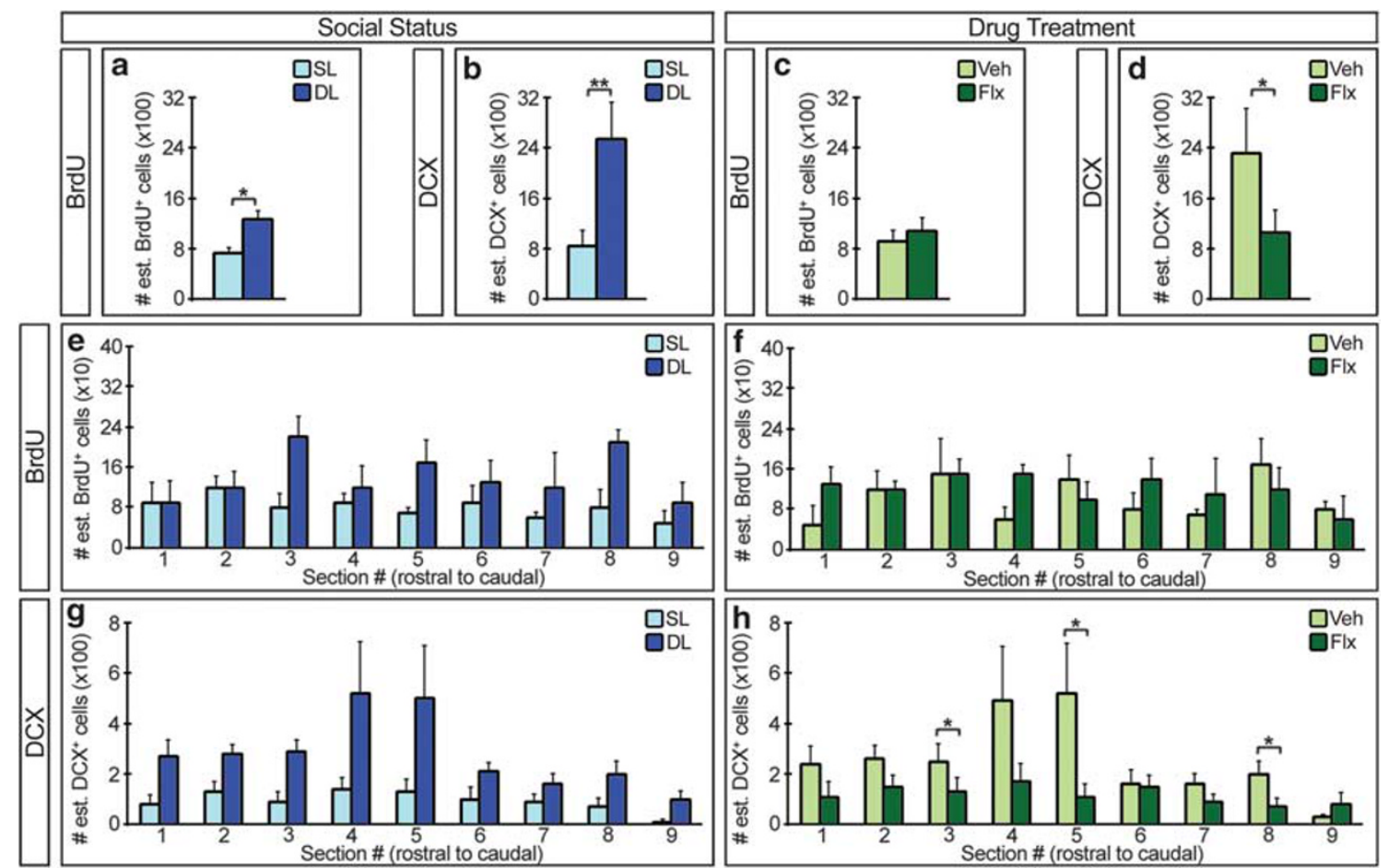

Figure 2 Effects of social status and drug treatment on adult cell proliferation and neurogenesis. Every 40th coronal section ( $1.6 \mathrm{~mm}$ apart) through the rostrocaudal extent of the right hippocampus of adult male baboons was stained for either bromodeoxyuridine (BrdU) or doublecortin (DCX) and the number of positively stained cells was quantified. Presented cell numbers were estimated by multiplying the number of quantified cells by 40 . (a and b) There are more BrdU- (a) and DCX-positive (b) cells in the dentate gyrus of dominant-like (DL) compared with subordinate-like (SL) animals. (c and d) Fluoxetine-treated baboons (Flx) have similar numbers of BrdU-positive cells (c) but significantly fewer DCX-positive cells (d) in the dentate gyrus compared with vehicle-treated baboons (Veh). (e and $f$ ) There is no significant interaction between social status (e) or antidepressant treatment ( $f$ ) and section number along the rostrocaudal axis for numbers of BrdU-positive cells. ( $g$ and $h$ ) There is no interaction between section number and social status ( $g$ ) for DCXpositive cells. However, a significant interaction exists between section number and drug treatment on numbers of DCX-positive cells (h), with significantly fewer cells in the third, fifth, and eighth sections of fluoxetine-treated animals. Mean \pm SEM; $n=4$ each group; * $p<0.05$; ** $p<0.0$ I for both parametric and non-parametric tests.

(Gould et al, 1998; Kozorovitskiy and Gould, 2004), we examined the effect of social status on neurogenesis in baboons. Animals were classified based on behavioral assessments (four DL and four SL). Dominant and subordinate behaviors were evident as soon as the baboons were housed in the same room. The most DL and SL baboons were quickly identified and their status remained stable over all their years in the colony. The three most DL baboons (Buddy, Condor, and Screech) displayed nearly entirely dominant behaviors, (including cage rattling to get attention from the caretaker or when another baboon was getting attention from the caretaker, aggressively reaching out of the cage to take treats or to grab the caretaker, and reaching into the adjoining baboon's cage; see MATERIALS AND METHODS for detailed list of behaviors) and rarely, if ever, exhibited subordinate behaviors. By contrast the three most SL baboons (Basie, DC, and Pennedy) displayed entirely subordinate behaviors. Two intermediate baboons were also readily identified who displayed a significant amount of both dominant and subordinate behaviors over the course of their time in the facility. These baboons would occasionally rattle the cage or demand attention but would also then be hesitant to come to the front of the cage to receive attention. While social status among the intermediate baboons fluctuated over time, they remained subordinate to the initially dominant animals and dominant to the initially subordinate baboons. At the behavioral assessment completed just before condition assignment, one of these animals was classified as DL (Dutch) and the other as SL (Grey) based on the fact that Dutch exhibited dominant behaviors more frequently than subordinate behaviors, whereas Grey exhibited subordinate behaviors more frequently than dominant ones. The number of both BrdUand DCX-expressing cells were significantly higher in DL compared with SL baboons (parametric-BrdU: $\mathrm{F}(1,5)=10.50, p=0.023 ; \quad \mathrm{DCX}: \mathrm{F}(1,5)=17.30, p=0.009$; non-parametric-BrdU: $\mathrm{F}(1,5)=16.84, \quad p=0.009 ; \quad \mathrm{DCX}$ : $\mathrm{F}(1,5)=27.22, p=0.003$; Figure $2 \mathrm{a}$ and $\mathrm{b})$.

To determine whether chronic antidepressant administration would potentiate adult neurogenesis in the baboon DG, animals were administered chronic oral fluoxetine or vehicle. Fluoxetine administration was confirmed, via postmortem quantification of hepatic fluoxetine and norfluoxetine, to result in levels corresponding to human therapeutic dose ranges (Johnson et al, 2007; Koran et al, 1996), whereas vehicle administration resulted in non-detectable levels of either fluoxetine or norfluoxetine. We found that the number of BrdU-positive cells was equivalent between fluoxetine- and vehicle-treated animals (parametric$\mathrm{F}(1,5)=0.92, \quad p=0.381 ; \quad$ non-parametric $-\mathrm{F}(1,5)=0.26$, $p=0.630$; Figure $2 \mathrm{c}$ ). Interestingly, fluoxetine treatment resulted in significantly fewer DCX-positive cells compared 

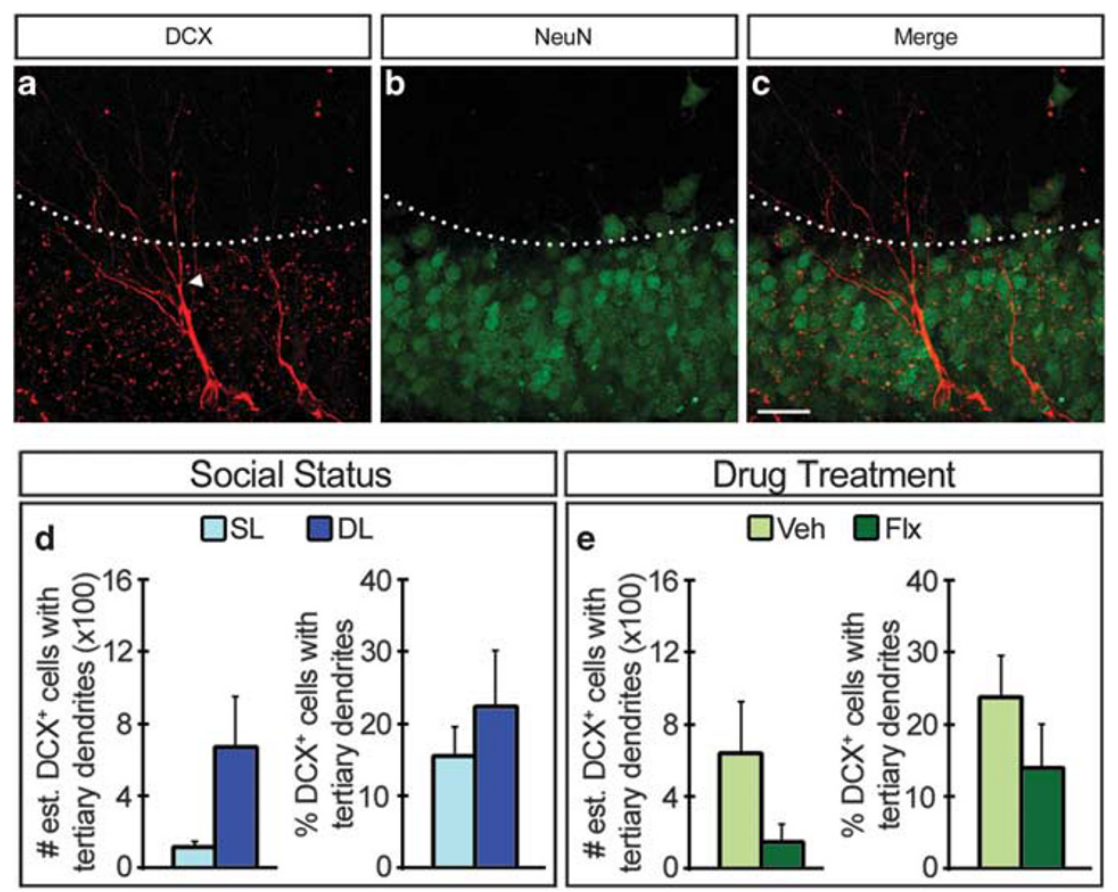

Figure 3 Effects of dominance and fluoxetine on maturation of adult-born neurons. $(a-c)$ Coronal section through a representative adult male baboon hippocampus stained for DCX ( $\mathrm{a}$ and $\mathrm{c}$ ) and $\mathrm{NeuN}(\mathrm{b}$ and $\mathrm{c}$ ). Arrowhead indicates tertiary branching of a DCX-positive neuron. Scale bar equals $25 \mu \mathrm{m}$. Dotted line denotes border of granule cell layer. (d) There is a trend for more DCX-positive cells with tertiary dendrites in DL compared with SL animals. However, there is no difference in the percentage of total DCX-positive cells with tertiary or higher order dendritic branching. (e) Fluoxetine treatment results in a trend toward fewer DCX-positive cells with tertiary dendrites. No effect is seen in the percentage of total DCX-positive cells with tertiary dendrites. Mean $\pm \mathrm{SEM} ; n=4$ each group.

with vehicle-treated animals (parametric- $\mathrm{F}(1,5)=9.47$, $p=0.028 ; \quad$ non-parametric $-\mathrm{F}(1,5)=13.89, \quad p=0.014$ Figure 2d). No significant interaction was seen between social status and drug treatment in either the number of BrdU- or DCX-positive cells (parametric-BrdU: $\mathrm{F}(1,4)=$ $0.01, p=0.920$; $\mathrm{DCX}: \mathrm{F}(1,4)=2.06, p=0.224$; non-parametric-BrdU: $\mathrm{F}(1,4)=0.22, p=0.662$; DCX: $\mathrm{F}(1,4)=0.00$, $p=1.000)$.

\section{Neurogenesis Along the Rostrocaudal Axis of the Baboon DG}

As the ventral (corresponding to the rostral in primates) pole of the hippocampus has been specifically implicated in the regulation of anxiety and depression-related behaviors in rodents (Fanselow and Dong, 2010), we sought to determine whether a rostrocaudal bias was present in our observed regulation of neurogenesis by social status and antidepressant treatment. There was no interaction between section number along the hippocampal rostrocaudal axis and either social status or drug treatment on the number of BrdU-positive cells or between section number and social status on the number of DCX-positive cells (Figure 2e-g, Supplementary Table S1). However, we found a significant interaction between section number and drug treatment on the number of DCX-positive cells (parametric$\mathrm{F}(8,32)=3.73, p=0.004$; non-parametric $-\mathrm{F}(8,32)=2.62$, $p=0.025)$. Post hoc comparisons revealed significant differences in the third $(p=0.047)$, fifth $(p=0.001)$, and eighth $(p=0.007)$ sections (Figure $2 \mathrm{~h}$ ).

\section{Maturity of Adult-Born Cells in the DG}

Antidepressants alter not only the generation of new cells in adulthood but also maturation of these adult-born neurons (David et al, 2009; Perera et al, 2011; Wang et al, 2008). As only DCX-positive cells with visible processes were quantified, we were unable to classify adult-born neurons using standard dendritic morphology criteria (Plumpe et al, 2006). We therefore examined the effects of social status or drug treatment on maturation of DCX-positive cells in the DG of baboons by quantifying the number of DCX-positive cells with tertiary and higher-order dendritic branching (Figure 3a-c; Wang et al, 2008). We observed a trend toward more DCX-positive cells with tertiary dendrites in DL compared with SL baboons (parametric- $\mathrm{F}(1,5)=6.51$, $p=0.051$; non-parametric $-\mathrm{F}(1,5)=2.58, p=0.169)$ as well as a trend in the opposite direction due to fluoxetine treatment (parametric- $\mathrm{F}(1,5)=5.16, p=0.072$; non-parametric $-\mathrm{F}(1,5)=5.81, p=0.061$; Figure $3 \mathrm{~d}$ and e). It is possible that these differences are simply due to an overall change in the absolute number of DCX-positive neurons rather than altered rates of differentiation. We therefore examined the percentage of DCX-positive neurons with tertiary or higher-order dendrites and indeed found no differences in these percentages between either DL and SL (parametric- $\mathrm{F}(1,5)=0.62, \quad p=0.466 ;$ non-parametric$\mathrm{F}(1,5)=0.76, p=0.422)$ or fluoxetine- and vehicle-treated baboons (parametric- $\mathrm{F}(1,5)=1.30, p=0.307$; non-parametric-F(1,5) =1.36, $p=0.297$; Figure $3 \mathrm{~d}$ and e). As an additional measure of dendritic complexity, all quantified DCX-positive cells were traced in order to determine total 

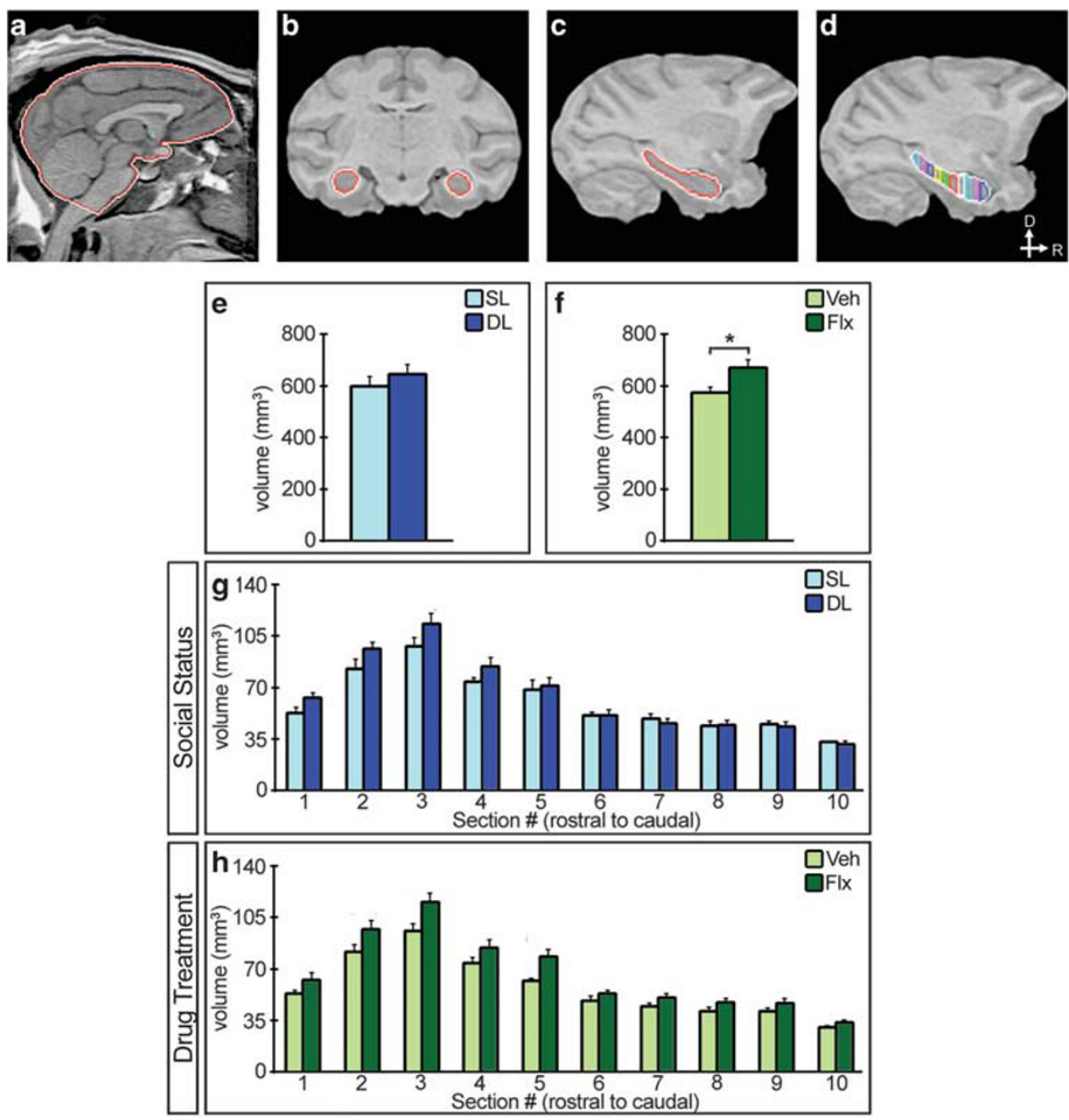

Figure 4 Effects of social status and drug treatment on baboon hippocampal volume. (a) Sagittal image of the magnetic resonance imaging (MRI) template of adult male baboon subjects. Rostral is to the right, dorsal to the top. Red line delineates the mask used for brain extraction of individual subject files. (b-d) Coronal (b) and sagittal (c and d) images of baboon brain template with hippocampus circumscribed in red (b and c) or virtually segmented into I0 similarly spaced coronal regions outlined in color (d). (e) There is no difference in whole hippocampal volume as imaged by MRI between DL and SL baboons. ( $f$ ) Whole hippocampal volume is significantly larger in animals treated with fluoxetine compared with animals administered vehicle. ( $\mathrm{g}$ and $\mathrm{h}$ ) There is no significant interaction between social status (g) or antidepressant treatment $(\mathrm{h})$ and section number along the rostrocaudal axis for in vivo hippocampal volume. Mean $\pm \mathrm{SEM} ; n=4$ each group; $* p<0.05$ for both parametric and non-parametric tests.

dendritic length, number of dendritic branches, and the average branch length per cell for each animal. No significant differences were seen between either DL and SL or fluoxetine- and vehicle-treated baboons (Supplementary Figure S2).

\section{In vivo Volumetric Analysis of the Baboon Hippocampus}

Neuroimaging studies in human and non-human primates have demonstrated a relationship between decreased hippocampal volume and depressive state (Czeh and Lucassen, 2007; Willard et al, 2011). Hippocampal volume is additionally correlated with antidepressant treatment in patients with MDD (Huang et al, 2013; Sheline et al, 2003). In order to determine whether any such relationship exists in baboons, structural MRIs were taken of all animals in the week before perfusion (Figure 4, Supplementary Figure $\mathrm{S} 1 \mathrm{e}, \mathrm{f})$. As there was no significant difference in volume between left and right hippocampus (data not shown), combined averaged volumes of both hemispheres were used for all analyses. Whereas we saw no difference in overall volume due to social status (parametric- $\mathrm{F}(1,5)=1.53$, $p=0.271 ; \quad$ non-parametric $-\mathrm{F}(1,5)=1.73, \quad p=0.245$; Figure 4e), fluoxetine-treated animals had a significantly larger hippocampus compared with vehicle-treated baboons (parametric- $\mathrm{F}(1,5)=6.80, \quad p=0.048$; non-parametric$\mathrm{F}(1,5)=9.42, p=0.028$; Figure $4 \mathrm{f})$. To examine whether this volumetric difference changed across the rostrocaudal axis, the whole hippocampus was virtually sliced into 10 coronal sections (Figure 4d). Although there appears to be a trend towards a rostral-specific effect of both social status and drug treatment on hippocampal volume, no significant interaction was seen between section number and either social status or drug treatment (Figure $4 \mathrm{~g}$ and $\mathrm{h}$ ).

\section{Volume of the DG GCL}

To determine whether any differences observed in cell proliferation, neurogenesis, or hippocampal volume in the 

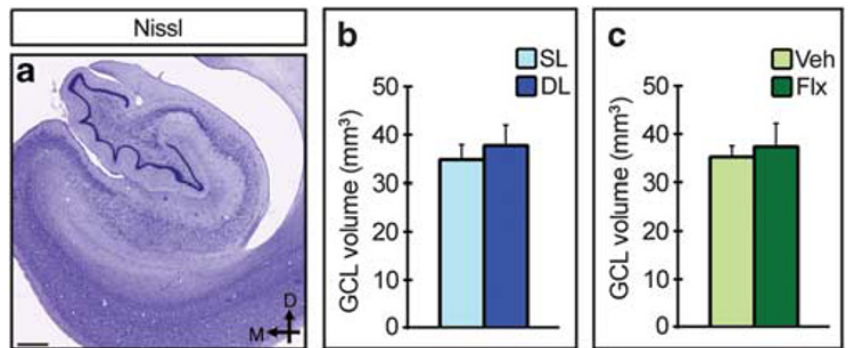

Figure 5 Dentate gyrus granule cell layer volume is independent of social status or drug treatment. (a) Coronal section of a representative adult male baboon hippocampus stained with cresyl violet. The granule cell layer (GCL) of the dentate gyrus is intensely labeled. Scale bar equals $1 \mathrm{~mm}$. Dorsal and medial are to the top and left, respectively. (b and c) There is no difference in the volume occupied by the GCL between $\mathrm{DL}$ and $\mathrm{SL}$ baboons (b) or between fluoxetine- and vehicle-treated animals (c). Mean \pm SEM; $n=4$ each group.

baboon were also reflected in an overall alteration in the absolute size of the DG, we quantified the total volume of the GCL of the DG in all animals. Intense Nissl staining of dentate granule cells allows for clear demarcation of the GCL (Figure 5a, Supplementary Figure S3). There were no significant differences in the total volume of the GCL between DL and SL baboons (parametric-F $(1,5)=0.28$, $p=0.620$; non-parametric- $\mathrm{F}(1,5)=0.25, \quad p=0.638)$ or between antidepressant- and vehicle-treated animals (parametric- $\mathrm{F}(1,5)=0.14, \quad p=0.721 ; \quad$ non-parametric$\mathrm{F}(1,5)=0.00, p=1.000$ ) (Figure $5 \mathrm{~b}$ and $\mathrm{c}$ ).

\section{DISCUSSION}

To our knowledge, this is the first complementary examination of adult hippocampal neurogenesis and in vivo MRIestimated volume in the baboon. We note that, due to the nature of the model system employed, our study represents a small number of samples. Thus, every effort has been made to analyze and interpret the data appropriately, including utilizing both parametric and non-parametric tests of significance. Our results suggest that hippocampal plasticity in the baboon, as measured by total volume or levels of adult neurogenesis, can be modulated by both social status and antidepressant treatment.

Given the timing of BrdU infusion 5 days before perfusion, our results do not allow for specific assessment of neurogenic precursor cells in the baboon DG. Future studies examining expression of additional proliferative markers such as Ki67 or PCNA (Kornack and Rakic, 1999; Perera et al, 2011) will assist in a finer dissection of the timeline of adult neurogenesis in the baboon. However, by concurrently examining the expression of DCX, we were able to estimate the number of immature adult-born neurons. The number of new neurons generated, although lower than that seen in birds and rodents, is consistent with that observed in other non-human primates (Kohler et al, 2011; Kornack and Rakic, 1999). Assuming a range of 5-9 million dentate granule cells in the primate (human and non-human) hippocampus (Seress, 2007), this number represents between 0.01 and $0.02 \%$ of the total hippocampal granule cell population, similar to percentages seen in other species (Kornack and Rakic, 1999; Spalding et al, 2013). Our results show that, similar to rodents (Kozorovitskiy and Gould, 2004), a DL hierarchical status is associated with a larger number of hippocampal adult-born neurons in the baboon. In contrast to previous work however (Perera et al, 2011), we do not see an effect of chronic antidepressant treatment on proliferation, and, surprisingly, observe that chronic fluoxetine treatment results in fewer immature neurons in the DG. Note, however, that previous work has also demonstrated that fluoxetine may have differing effects on neurogenesis depending on the age and sex of subjects studied (Couillard-Despres et al, 2009; Hodes et al, 2009; Lucassen et al, 2010).

Alternately, it is possible that fluoxetine accelerated the maturation of young neurons as has been observed in mice (David et al, 2009; Wang et al, 2008). Such an accelerated maturation might result in a more rapid loss of DCX expression, and thus lower numbers of DCX-positive cells. As these more mature neurons no longer express DCX, increased maturation may not have been reflected in our analysis of DCX-positive cells. We note, however, that our analysis may underestimate the dendritic complexity of DCX-positive cells due to the thickness of our examined sections and it will therefore be important to examine thicker sections in future studies in order to perform a full dendritic analysis of these adult-born dentate granule cells. Possibly related to this phenomenon is the observation that under baseline conditions, maturation of adult-born granule cells is slower in primates than in rodents (Kohler et al, 2011). Unlike the situation, in mice (Wang et al, 2008) we do not see co-expression of DCX and NeuN, precluding the ability to further dissect how fluoxetine administration may alter maturation of adult-born neurons. In future studies, it will be important to determine the impact of antidepressants on neural differentiation by tracking the survival of adult-born neurons over time using either BrdU or retroviral-mediated fluorescent labeling (van Praag et al, 2002) and examining subsequent expression of later markers of neuronal maturation including NeuN, calretinin, and calbindin (Kobayashi et al, 2010).

\section{Relationship between Neurogenesis and Hippocampal Volume}

In vivo hippocampal volume is known to be negatively and positively correlated with MDD and antidepressant administration, respectively (Czeh and Lucassen, 2007). In untreated depressed patients, in vivo hippocampal volume was smaller than in antidepressant-treated patients (Huang et al, 2013) and was negatively correlated with time spent depressed while being untreated (Sheline et al, 2003), suggesting a positive effect of antidepressant treatment on whole hippocampal volume. In post-mortem samples, DG volume (including both molecular and granule cell layers) was larger in patients treated with antidepressants (Boldrini et al, 2009). DG volume also correlated with both neural progenitor cell number and DG vascularization (Boldrini et al, 2012). Given the difficulty in assessing neurogenesis in patient populations (Couillard-Despres and Aigner, 2011), however, a definitive relationship between changes in neurogenesis and hippocampal volume in humans has yet to be delineated (Czeh and Lucassen, 2007). Our study 
allows for the first examination of whether a correlation exists between measures of hippocampal volume and adult hippocampal neurogenesis in the baboon. We show that, whereas a 30-day course of antidepressant treatment in two socially dominant and two socially subordinate animals does not alter the number of proliferating cells in the hippocampal DG, overall hippocampal volume is greater. In fact, we found no correlation between overall hippocampal volume and total number of either BrdU- or DCX-positive cells (Supplementary Figure S4), although this study is underpowered to definitively test for a relationship. We recognize that, as we were unable to measure total brain volume, an unfortunate limitation of our study is an inability to exclude the possibility that our observed fluoxetine-induced increase in hippocampal volume is simply due to increased total brain volume. However, it is worth noting that the contribution of adult-born dentate granule cells to whole hippocampal volume is small and that the largest fraction of hippocampal volume comes from the dendrites of principal neurons that have been shown to be influenced by stress (McEwen, 1999). Further examination is thus required to determine whether the effect of fluoxetine on hippocampal volume in our study and others (Huang et al, 2013; Sheline et al, 2003) may in fact be due to changes in neuropil and vascularization. It will also be interesting, in future studies, to examine effects of antidepressants on total brain and hippocampal volume in a within-subject manner in order to assess percentage volumetric changes from baseline.

\section{Social Regulation of Hippocampal Plasticity}

Consistent with previous studies (Kozorovitskiy and Gould, 2004), our results indicate a positive correlation between DL social status and adult hippocampal neurogenesis, and, assuming that low social status is associated with greater stress, are consistent with work demonstrating that stress decreases cell proliferation and survival of adult-born granule cells (David et al, 2009; Gould et al, 1998; Pham et al, 2003). However, it has been shown that stress and social rank are not always positively correlated and that in fact, plasma cortisol levels, used as a marker of stress, can be higher in dominant individuals in certain instances (Abbott et al, 2003). Note that the relation between stress and social hierarchy is dependent on the strain and social organization of the non-human primate studied. As a relative indicator of stress levels, we examined hepatic expression of genes related to local cortisol production (NR3C1-glucocorticoid receptor; PCK1-phosphoenolpyruvate carboxykinase 1; and HSD11B1-hydroxysteroid (11 $\beta$ ) dehydrogenase 1) but found no significant differences in expression levels. Interestingly, although behavioral and physiological differences have been characterized between non-human primates that are socially stressed and those that are depressed, socially stressed animals have an increased tendency to become depressed compared with non-stressed animals (Shively and Willard, 2012). We recognize that our baboons are singly housed and although we have classified them as DL or SL based on behaviors that parallel those in the wild, our estimates are a model of real world behavior. It will therefore be interesting, in future studies, to examine animals in a field setting in order to correlate social rank, stress, depression, and hippocampal plasticity in these primates.

\section{Is Adult Hippocampal Neurogenesis Relevant in Humans?}

There is no doubt that adult-born neurons have a critical functional role in birds and rodents. However, the $\sim 15$-fold relative decrease in the generation of cells in non-human primates (Kohler et al, 2011) raises the important question of whether adult neurogenesis is functionally relevant in humans (Goritz and Frisen, 2012; Snyder and Cameron, 2012). Recent work has suggested that some of these discrepancies may be explained by differences in the age of animals studied (Ben Abdallah et al, 2010), and that levels of neurogenesis in middle-aged humans are actually similar to those in middle-aged rodents (Spalding et al, 2013). Given a 30 -year average lifespan for baboons in the wild, our subjects likely represent animals in young adulthood and it will be interesting to examine hippocampal plasticity in baboons of different ages in future studies. It should be noted, however, that even in rodents, the number of adultgenerated neurons comprises a small percentage of the total number of neurons and yet this small neural population significantly influences a diverse set of behaviors (Snyder and Cameron, 2012). It has also been suggested that the small population of adult-born neurons exerts a large behavioral effect via inhibitory modulation of the mature neural network (Sahay et al, 2011). Given the increased time to maturation in primates (Kohler et al, 2011), it is possible that these adult-born neurons may even have increased functional relevance in primates. Following the pivotal study characterizing BrdU incorporation in humans (Eriksson et al, 1998), there have been limited studies examining markers of proliferation or neural stem cells in the DG of healthy and diseased patient populations (Boldrini et al, 2012; Boldrini et al, 2009; Knoth et al, 2010; Lucassen et al, 2010; Monje et al, 2007; Spalding et al, 2013). Increasing the body of knowledge examining the extent of neurogenesis and its functional role in both human and non-human primates (and the number of species in which this phenomenon is characterized) will allow for a better understanding of whether and how to target neurogenesis in human patients.

\section{FUNDING AND DISCLOSURE}

JS worked for a digital histopathology company, Sinq Systems; however, the company was not involved with any portion of the study. She is currently located at Pfizer Neuroscience. RH receives compensation as a consultant for Lundbeck, Roche, and Servier. The remaining authors declare no conflict of interest.

\section{ACKNOWLEDGEMENTS}

We gratefully acknowledge the assistance from the following individuals: animal care-A Ramirez, J Willi, G Asfaw, J Castrillon; imaging-F Liu, B Avants, P Cook, J Gee, M Baxter, P Rapp; histology $-M$ Underwood, M Douglass, $Y$ Liu; gene expression-J Kral, Z Donaldson; biochemistry- 
R Suckow, T Cooper, T Su, X Wang. Financial support was provided by: T32-MH015174/NIMH (MVW); AG-016765/ NIA, Intramural Research Program of the National Institute of Aging (JS); P50-MH62185/NIMH (VA); R01-MH83862/ NIMH, R21-94888/NIMH, Diane Goldberg Foundation (MB); R01-DA004130/NIDA, K05-DA031749/NIDA (RWF); R37-MH068542/NIMH, NYSTEM C024330, and Hope for Depression Research Foundation (RH) grants.

\section{REFERENCES}

Abbott DH, Keverne EB, Bercovitch FB, Shively CA, Mendoza SP, Saltzman W et al (2003). Are subordinates always stressed? A comparative analysis of rank differences in cortisol levels among primates. Horm Behav 43: 67-82.

Avants BB, Epstein CL, Grossman M, Gee JC (2008). Symmetric diffeomorphic image registration with cross-correlation: evaluating automated labeling of elderly and neurodegenerative brain. Med Image Anal 12: 26-41.

Avants BB, Tustison NJ, Song G, Cook PA, Klein A, Gee JC (2011). A reproducible evaluation of ANTs similarity metric performance in brain image registration. Neuroimage 54: 2033-2044.

Avants BB, Yushkevich P, Pluta J, Minkoff D, Korczykowski M, Detre J et al (2010). The optimal template effect in hippocampus studies of diseased populations. Neuroimage 49: 2457-2466.

Ben Abdallah NM, Slomianka L, Vyssotski AL, Lipp HP (2010). Early age-related changes in adult hippocampal neurogenesis in C57 mice. Neurobiol Aging 31: 151-161.

Boldrini M, Hen R, Underwood MD, Rosoklija GB, Dwork AJ, Mann JJ et al (2012). Hippocampal angiogenesis and progenitor cell proliferation are increased with antidepressant use in major depression. Biol Psychiatry 72: 562-571.

Boldrini M, Underwood MD, Hen R, Rosoklija GB, Dwork AJ, John Mann J et al (2009). Antidepressants increase neural progenitor cells in the human hippocampus. Neuropsychopharmacology 34: 2376-2389.

Cameron HA, Gould E (1994). Adult neurogenesis is regulated by adrenal steroids in the dentate gyrus. Neuroscience 61: 203-209.

Conover WJ, Iman RL (1981). Rank transformations as a bridge between parametric and nonparametric statistics. Am Stat 35: 124-129.

Couillard-Despres S, Aigner L (2011). In vivo imaging of adult neurogenesis. Eur J Neurosci 33: 1037-1044.

Couillard-Despres S, Winner B, Schaubeck S, Aigner R, Vroemen $\mathrm{M}$, Weidner $\mathrm{N}$ et al (2005). Doublecortin expression levels in adult brain reflect neurogenesis. Eur J Neurosci 21: 1-14.

Couillard-Despres S, Wuertinger C, Kandasamy M, Caioni M, Stadler K, Aigner R et al (2009). Ageing abolishes the effects of fluoxetine on neurogenesis. Mol Psychiatry 14: 856-864.

Czeh B, Lucassen PJ (2007). What causes the hippocampal volume decrease in depression? Are neurogenesis, glial changes and apoptosis implicated? Eur Arch Psychiatry Clin Neurosci 257: 250-260.

David DJ, Samuels BA, Rainer Q, Wang JW, Marsteller D, Mendez I et al (2009). Neurogenesis-dependent and -independent effects of fluoxetine in an animal model of anxiety/depression. Neuron 62: 479-493.

Davis R, Huffman RD (1968). A Stereotaxic Atlas of the Brain of the Baboon (Papio). University of Texas Press: Austin, TX, xvii, 134 p. chiefly illus.pp.

Deng W, Aimone JB, Gage FH (2010). New neurons and new memories: how does adult hippocampal neurogenesis affect learning and memory? Nat Rev Neurosci 11: 339-350.

Denny CA, Burghardt NS, Schachter DM, Hen R, Drew MR (2012). 4- to 6-week-old adult-born hippocampal neurons influence novelty-evoked exploration and contextual fear conditioning. Hippocampus 22: 1188-1201.

Dranovsky A, Picchini AM, Moadel T, Sisti AC, Yamada A, Kimura S et al (2011). Experience dictates stem cell fate in the adult hippocampus. Neuron 70: 908-923.

Eriksson PS, Perfilieva E, Bjork-Eriksson T, Alborn AM, Nordborg C, Peterson DA et al (1998). Neurogenesis in the adult human hippocampus. Nat Med 4: 1313-1317.

Fanselow MS, Dong HW (2010). Are the dorsal and ventral hippocampus functionally distinct structures? Neuron 65: 7-19.

Foltin RW (2011). Consumption of palatable food decreases the anorectic effects of serotonergic, but not dopaminergic drugs in baboons. Physiol Behav 103: 493-500.

Goritz C, Frisen J (2012). Neural stem cells and neurogenesis in the adult. Cell Stem Cell 10: 657-659.

Gould E, Tanapat P, McEwen BS, Flugge G, Fuchs E (1998). Proliferation of granule cell precursors in the dentate gyrus of adult monkeys is diminished by stress. Proc Natl Acad Sci USA 95: 3168-3171.

Hodes GE, Yang L, Van Kooy J, Santollo J, Shors TJ (2009). Prozac during puberty: distinctive effects on neurogenesis as a function of age and sex. Neuroscience 163: 609-617.

Huang Y, Coupland NJ, Lebel RM, Carter R, Seres P, Wilman AH et al (2013). Structural changes in hippocampal subfields in major depressive disorder: a high-field magnetic resonance imaging study. Biol Psychiatry 74: 62-68.

Johnson RD, Lewis RJ, Angier MK (2007). The distribution of fluoxetine in human fluids and tissues. J Anal Toxicol 31: 409-414.

Kempermann G, Kuhn HG, Gage FH (1997). More hippocampal neurons in adult mice living in an enriched environment. Nature 386: 493-495.

Knoth R, Singec I, Ditter M, Pantazis G, Capetian P, Meyer RP et al (2010). Murine features of neurogenesis in the human hippocampus across the lifespan from 0 to 100 years. PLoS One 5: e8809.

Kobayashi K, Ikeda Y, Sakai A, Yamasaki N, Haneda E, Miyakawa $\mathrm{T}$ et al (2010). Reversal of hippocampal neuronal maturation by serotonergic antidepressants. Proc Natl Acad Sci USA 107: 8434-8439.

Kohler SJ, Williams NI, Stanton GB, Cameron JL, Greenough WT (2011). Maturation time of new granule cells in the dentate gyrus of adult macaque monkeys exceeds six months. Proc Natl Acad Sci USA 108: 10326-10331.

Koran LM, Cain JW, Dominguez RA, Rush AJ, Thiemann S (1996). Are fluoxetine plasma levels related to outcome in obsessivecompulsive disorder? Am J Psychiatry 153: 1450-1454.

Kornack DR, Rakic P (1999). Continuation of neurogenesis in the hippocampus of the adult macaque monkey. Proc Natl Acad Sci USA 96: 5768-5773.

Kozorovitskiy Y, Gould E (2004). Dominance hierarchy influences adult neurogenesis in the dentate gyrus. J Neurosci 24: 6755-6759.

Longair MH, Baker DA, Armstrong JD (2011). Simple Neurite Tracer: open source software for reconstruction, visualization and analysis of neuronal processes. Bioinformatics 27: 2453-2454.

Lucassen PJ, Stumpel MW, Wang Q, Aronica E (2010). Decreased numbers of progenitor cells but no response to antidepressant drugs in the hippocampus of elderly depressed patients. Neuropharmacology 58: 940-949.

Malberg JE, Eisch AJ, Nestler EJ, Duman RS (2000). Chronic antidepressant treatment increases neurogenesis in adult rat hippocampus. J Neurosci 20: 9104-9110.

McEwen BS (1999). Stress and hippocampal plasticity. Annu Rev Neurosci 22: 105-122.

Ming GL, Song H (2011). Adult neurogenesis in the mammalian brain: significant answers and significant questions. Neuron 70: 687-702. 
Monje ML, Vogel H, Masek M, Ligon KL, Fisher PG, Palmer TD (2007). Impaired human hippocampal neurogenesis after treatment for central nervous system malignancies. Ann Neurol 62: 515-520.

Perera TD, Dwork AJ, Keegan KA, Thirumangalakudi L, Lipira CM, Joyce $\mathrm{N}$ et al (2011). Necessity of hippocampal neurogenesis for the therapeutic action of antidepressants in adult nonhuman primates. PLoS One 6: e17600.

Pham K, Nacher J, Hof PR, McEwen BS (2003). Repeated restraint stress suppresses neurogenesis and induces biphasic PSA-NCAM expression in the adult rat dentate gyrus. Eur $J$ Neurosci 17: 879-886.

Plumpe T, Ehninger D, Steiner B, Klempin F, Jessberger S, Brandt $M$ et al (2006). Variability of doublecortin-associated dendrite maturation in adult hippocampal neurogenesis is independent of the regulation of precursor cell proliferation. BMC Neurosci 7: 77.

Rakic P (2002). Neurogenesis in adult primate neocortex: an evaluation of the evidence. Nat Rev Neurosci 3: 65-71.

Sahay A, Hen R (2007). Adult hippocampal neurogenesis in depression. Nat Neurosci 10: 1110-1115.

Sahay A, Scobie KN, Hill AS, O'Carroll CM, Kheirbek MA, Burghardt NS et al (2011). Increasing adult hippocampal neurogenesis is sufficient to improve pattern separation. Nature 472: $466-470$.

Schindelin J, Arganda-Carreras I, Frise E, Kaynig V, Longair M, Pietzsch $\mathrm{T}$ et al (2012). Fiji: an open-source platform for biological-image analysis. Nat Methods 9: 676-682.

Seress L (2007). Comparative anatomy of the hippocampal dentate gyrus in adult and developing rodents, non-human primates and humans. Prog Brain Res 163: 23-41.

Shamy JL, Buonocore MH, Makaron LM, Amaral DG, Barnes CA, Rapp PR (2006). Hippocampal volume is preserved and fails to predict recognition memory impairment in aged rhesus monkeys (Macaca mulatta). Neurobiol Aging 27: 1405-1415.
Shamy JL, Habeck C, Hof PR, Amaral DG, Fong SG, Buonocore $\mathrm{MH}$ et al (2011). Volumetric correlates of spatiotemporal working and recognition memory impairment in aged rhesus monkeys. Cereb Cortex 21: 1559-1573.

Sheline YI, Gado MH, Kraemer HC (2003). Untreated depression and hippocampal volume loss. Am J Psychiatry 160: 1516-1518.

Shively CA, Willard SL (2012). Behavioral and neurobiological characteristics of social stress versus depression in nonhuman primates. Exp Neurol 233: 87-94.

Snyder JS, Cameron HA (2012). Could adult hippocampal neurogenesis be relevant for human behavior? Behav Brain Res 227: 384-390.

Spalding KL, Bergmann O, Alkass K, Bernard S, Salehpour M, Huttner HB et al (2013). Dynamics of hippocampal neurogenesis in adult humans. Cell 153: 1219-1227.

Suckow RF, Zhang MF, Cooper TB (1992). Sensitive and selective liquid-chromatographic assay of fluoxetine and norfluoxetine in plasma with fluorescence detection after precolumn derivatization. Clin Chem 38: 1756-1761.

Surget A, Saxe M, Leman S, Ibarguen-Vargas Y, Chalon S, Griebel G et al (2008). Drug-dependent requirement of hippocampal neurogenesis in a model of depression and of antidepressant reversal. Biol Psychiatry 64: 293-301.

van Praag H, Schinder AF, Christie BR, Toni N, Palmer TD, Gage FH (2002). Functional neurogenesis in the adult hippocampus. Nature 415: 1030-1034.

Wang JW, David DJ, Monckton JE, Battaglia F, Hen R (2008). Chronic fluoxetine stimulates maturation and synaptic plasticity of adult-born hippocampal granule cells. J Neurosci 28: $1374-1384$.

Willard SL, Daunais JB, Cline JM, Shively CA (2011). Hippocampal volume in postmenopausal cynomolgus macaques with behavioral depression. Menopause 18: 582-586.

Supplementary Information accompanies the paper on the Neuropsychopharmacology website (http://www.nature.com/npp) 